The Annals of Probability

2002, Vol. 30, No. 2, 802-825

\title{
ON STOCHASTIC DIFFERENTIAL EQUATIONS DRIVEN BY A CAUCHY PROCESS AND OTHER STABLE LÉVY MOTIONS
}

\author{
By Pio ANDREA ZANZOTTO
}

\section{Università di Udine} tions

We consider the class of one-dimensional stochastic differential equa-

$$
d X_{t}=b\left(X_{t^{-}}\right) d Z_{t}, \quad t \geq 0,
$$

where $b$ is a Borel measurable real function and $Z$ is a strictly $\alpha$-stable Lévy process $(0<\alpha \leq 2)$. Weak solutions are investigated improving previous results of the author in various ways.

In particular, for the equation driven by a strictly 1-stable Lévy process, a sufficient existence condition is proven.

Also we extend the weak existence and uniqueness exact criteria due to Engelbert and Schmidt for the Brownian case (i.e., $\alpha=2$ ) to the class of equations with $\alpha$ such that $1<\alpha \leq 2$. The results employ some representation properties with respect to strictly stable Lévy processes.

0. Introduction. In this paper we consider the one-dimensional stochastic differential equation

$$
X_{t}=X_{0}+\int_{] 0, t]} b\left(X_{s^{-}}\right) d Z_{s}, \quad t \geq 0,
$$

where $X_{0}$ has an arbitrary distribution $\eta$ on $\mathbb{R}, b$ is a Borel measurable real function and $Z$ denotes a strictly $\alpha$-stable Lévy process, $0<\alpha \leq 2$. Moreover, when considering the above equation driven by $Z$ with parameter $\alpha \neq 2$, here we always adopt the following assumption:

\section{(A) Either $Z$ is symmetric or $b$ is nonnegative.}

For the above class of equations with $\alpha \neq 1$ and $Z$ symmetric, weak solutions have already been investigated by the author in an earlier paper [18]. Pursuing that study, results which were obtained there are improved here from various points of view.

This is made possible using some representation properties with respect to strictly stable Lévy processes in [19] as a key tool.

First, here we extend the weak existence and uniqueness exact criteria of Engelbert and Schmidt for (1) driven by Brownian motion (i.e., $Z$ with $\alpha=2$ ), to the class of the above equations driven by $Z$ with parameter $\alpha, 1<\alpha \leq 2$.

Received March 1999; revised August 2001.

AMS 2000 subject classifications. 60H10, 60J30.

Key words and phrases. Strictly $\alpha$-stable Lévy processes, Cauchy process, stochastic differential equations, weak existence, "local" existence, time change, quadratic pure-jump semimartingales, representation, stable integrals. 
More precisely, for a fixed $\alpha, 1<\alpha \leq 2$, define the singularity set

$$
I(\alpha)=\left\{x \in \mathbb{R}: \int_{x^{-}}^{x^{+}}|b(y)|^{-\alpha} d y=\infty\right\}
$$

and let $N$ denote the set of zeros of $b$. Recall the Engelbert-Schmidt result:

TheOREM ([4], Theorems 1, 2). Consider (1) driven by $Z$ with $\alpha=2$ and arbitrary initial distribution. Then weak existence holds if and only if $I(2) \subset N$. In that case uniqueness in law holds for every initial distribution if and only if $I(2)=N$.

(For a good exposition, see also [9], Theorem (20.1), or [10], Theorems 5.4, 5.5.)

The main result we obtain here is the following (see Theorems 2.2, 2.6 below):

THEOREM. Assumption (A) being in force, consider (1) driven by $Z$ with parameter $\alpha, 1<\alpha \leq 2$. Then weak existence holds for every initial distribution if and only if $I(\alpha) \subset N$. In that case uniqueness in law holds for every initial distribution if and only if $I(\alpha)=N$.

In the previous paper [18] we investigated nontrivial solutions of (1), that is, solutions which are to move away from the initial value with probability greater than 0: for the same case with $\alpha, 1<\alpha \leq 2$, the main result obtained there was the following, to be compared with the above theorem:

THEOREM ([18], (2.32)). Consider (1) driven by a symmetric $Z$ with $\alpha$, $1<\alpha \leq 2$. Then the following properties are equivalent:

(a) For every $x \in \mathbb{R}$ there exists a nontrivial solution starting from $x$.

(b) Function $|b|^{-\alpha}$ is locally integrable.

Such an existence criterion is clearly restrictive for the coefficient $b$, as involving the condition that $I(\alpha)$ be empty: see the examples at the end for simple cases where it is not verified.

Concerning nontrivial solutions with fixed initial point $x$, sufficient existence results in [18] were based on condition $(\mathrm{H})(x)$; see Definition 3.1 below. It turns out that such a condition can be used also in the case $\alpha=1$; so, combining a representation property by means of a 1-stable motion ([19], Theorem 1 and Corollary) with the occupation time measure property expressed in Proposition 1.1(b) below, we show that a nontrivial solution starting from $x$ exists if $b$ satisfies condition $(\mathrm{H})(x)$. See Theorem 3.3. This result extends Theorem (2.5) in [18] mainly including the case $\alpha=1$ (so also a first answer is given to a question proposed by one of the referees of [18]).

Condition $(\mathrm{H})$ is related to integrability conditions on $b$ alone: see Proposition 3.7. In particular, by combining that proposition with Theorem 3.3 the following results are deduced (see Theorems 3.8, 3.9): 
THEOREM. Consider (1) driven by the symmetric Cauchy process [resp. (1) with a nonnegative $b$, driven by a 1-stable Lévy motion with nonvanishing drift]. Assume that there exists a real number $\delta>1$ such that $|b|^{-\delta}$ is locally integrable. Then for any law $\eta$ there exists a nontrivial solution with initial distribution $\eta$.

THEOREM. Consider (1) in the case $0<\alpha<1$. Assume that (A) holds and there exists a real number $\delta>1$ such that $|b|^{-\delta}$ is locally integrable. Moreover there exists $U>0$ such that $\lambda\left(B_{U}\right)<\infty$, where $B_{U}=\{x \in \mathbb{R}:|b(x)|>U\}$. Then, for any law $\eta$ on $\mathbb{R}$, there exists a nontrivial solution with initial distribution $\eta$.

As to condition $(\mathrm{H})$ when $1<\alpha \leq 2$, improving the above-recalled Theorems (2.32) and (3.17) in [18], its meaning is completely clarified in Theorem 3.15 below. In particular, the existence of an $x_{0}$ such that $b$ satisfies $H\left(x_{0}\right)$ is equivalent to local integrability of $|b|^{-\alpha}$ (and thus to the existence of a nontrivial solution starting from every $x$ ).

It follows that $(\mathrm{H})(x)$ is not necessary for the existence of a nontrivial solution starting from $x$, as also Example 3.16 shows. However, we still consider $(\mathrm{H})(x)$ mainly in connection with its "localized" version, here denoted $(\mathrm{LH})(x)$ (see Definition 3.2), since results based on the latter condition go back to other involving $(\mathrm{H})(x)$. Such a condition as $(\mathrm{LH})(x)$ seems to be suited for the study of nontrivial "local" solutions starting from $x$, a process being termed a solution on an interval containing $x$ ("local" solution) if it solves the equation up to the first exit time of the interval (cf. Section 3).

As a sufficient condition for the existence of nontrivial local solutions starting from $x$, (LH) $(x)$ completely unifies the cases of (1) driven by $Z$ 's with different $\alpha$, $0<\alpha \leq 2$ (see Theorem 3.10):

THEOREM. Assumption (A) being in force, consider (1) driven by $Z$ with $0<\alpha \leq 2$. Assume $x$ to be such that $b$ satisfies condition $(\mathrm{LH})(x)$. Then there exists a nontrivial "local" solution starting from $x$.

When $1<\alpha \leq 2,(\mathrm{LH})(x)$ is also necessary for the existence of such solutions: see Corollary 3.13. When $0<\alpha \leq 1$, we do not know if a similar necessity property holds for $(\mathrm{LH})(x)$ : however, from examples it seems that condition $(\mathrm{LH})(x)$ is not far from a necessary one in this case also.

0.1. Preliminaries. General terminology and notation about processes used in [18] are in force also here. In particular, all the stochastic bases $\mathbb{B}=$ $(\Omega, \mathcal{F}, \mathbb{F}, P), \mathbb{F}=\left(\mathscr{F}_{t}\right)_{t \geq 0}$, we consider are supposed to satisfy "the usual conditions" of completeness and right-continuity and all the stochastic processes to be considered are assumed to be real-valued and to have all the sample paths in $\mathbb{D}=\mathbb{D}\left(\mathbb{R}_{+}, \mathbb{R}\right)$, the space of the càdlàg mappings from $\mathbb{R}_{+}=[0,+\infty[$ into $\mathbb{R}$. By 
the notation $(\mathbb{D}, \mathscr{D})$ we denote space $\mathbb{D}$ with the Skorokhod topology, equipped with the $\sigma$-algebra $\mathscr{D}$ of Borel sets in this topology (cf. [7], Chapter 6).

For a process $X=\left(X_{t}\right)_{t \geq 0}$ defined on $(\Omega, \mathcal{F}, \mathbb{F}, P)$, we write $(X, \mathbb{F})$ to mean that $X$ is $\mathbb{F}$-adapted; however, we suppress the " $\mathbb{F}$ " whenever its appearance is not essential.

Also the usual convention $0 \cdot \infty=0 / 0=0$ is adopted. Throughout the paper the notation $Z$ will denote a strictly $\alpha$-stable Lévy process, $0<\alpha \leq 2$. The case when $Z$ is a pure drift will be implicitly excluded.

To be more precise, the notation $(Z, \mathbb{F})$ denotes a process defined on a basis $\mathbb{B}=(\Omega, \mathcal{F}, \mathbb{F}, P)$ and having any initial distribution such that, for all $0 \leq s<t$, $\vartheta \in \mathbb{R}$, the following hold:

1. when $0<\alpha<2, \alpha \neq 1$,

$$
\mathbb{E}\left[\exp \left\{i \vartheta\left(Z_{t}-Z_{s}\right)\right\} \mid \mathcal{F}_{s}\right]=\exp \left\{-k(t-s)|\vartheta|^{\alpha}\left(1-i \beta \frac{\vartheta}{|\vartheta|} \tan \left(\pi \frac{\alpha}{2}\right)\right)\right\},
$$

where $k>0$ and $\beta \in[-1,1]$ ( $\beta$ is called the "skewness" parameter);

2. when $\alpha=2$, the above formula holds with $\alpha=2$ and $\beta=0$ (so a strictly

2-stable Lévy process is proportional to a standard Brownian motion);

3. when $\alpha=1$,

$$
\mathbb{E}\left[\exp \left\{i \vartheta\left(Z_{t}-Z_{s}\right)\right\} \mid \mathcal{F}_{s}\right]=\exp \{-(t-s)(k|\vartheta|-i \gamma \vartheta)\},
$$

where $\gamma \in \mathbb{R}$ is the drift coefficient and $k>0$.

If $\alpha=1$ and in the latter formula $\gamma=0,(Z, \mathbb{F})$ is termed a Cauchy process.

For the sake of brevity, in the sequel we omit the adjective "strictly."

Moreover, when the initial distribution of $Z$ is not explicitly mentioned, it will be always understood that $Z_{0}=0$ a.s.; that is, simply saying $Z$ is an $\alpha$-stable Lévy process will imply $Z_{0}=0$ a.s.; recall that in such a case, $Z$ is $1 / \alpha$-selfsimilar. Note that such processes are also called $\alpha$-stable Lévy motions (cf. [14], Definition 7.5.1, page 349), so here we sometimes also employ such a term to denote these objects.

We denote by $\sigma(\omega, t, \cdot)$ the occupation time measure of $Z$, that is, the measure defined as follows: for all Borel sets $A$ in $\mathbb{R}$ and all $\omega, t \geq 0$,

$$
\sigma(\omega, t, A)=\int_{0}^{t} \mathbb{1}_{A}\left(Z_{S}(\omega)\right) d s=\lambda\left(\left\{s \leq t: Z_{s}(\omega) \in A\right\}\right),
$$

$\lambda$ denoting Lebesgue measure, as always in the following.

As is well known, $Z$ has a local time if and only if $1<\alpha \leq 2$; by local time we mean here an occupation time density, that is, a function $L(\omega, t, y)$ such that, $P$-a.s.,

$$
\sigma(t, A)=\int_{\mathbb{R}} \mathbb{1}_{A}(y) L(t, y) d y
$$


for any $t$ and any Borel set $A$. Also, a version of $L(\omega, t, y)$ exists which is jointly measurable in $(\omega, t, y)$. Moreover $(t, y) \mapsto L(\omega, t, y)$ can be chosen to be jointly continuous for all $\omega$. In the sequel we always employ a version of the local time $L$ satisfying these regularity conditions.

1. Some properties of strictly $\boldsymbol{\alpha}$-stable Lévy processes. Let $Z$ denote an $\alpha$-stable Lévy process, $0<\alpha \leq 2$, on $(\Omega, \mathcal{F}, \mathbb{F}, P)$. (Recall the assumptions in the previous section.)

The following property completes Proposition (1.3) in [18] including the case $\alpha=1$ (also, when $0<\alpha<2, \alpha \neq 1$, the symmetry assumption in [18] is removed).

PROPOSITION 1.1. Let $\sigma$ denote the occupation time measure (0.1) of Z:

(a) Assume $0<\alpha<1$. Then we have

$$
P\left(\left\{\lim _{t \rightarrow \infty} \sigma(t, A)<\infty\right\}\right)=1
$$

for every Borel set $A$ such that $\int_{A}|y|^{\alpha-1} d y<\infty$ [thus for every Borel set $A$ such that $\lambda(A)<\infty]$.

(b) Assume $1 \leq \alpha \leq 2$. Then we have

$$
P\left(\left\{\lim _{t \rightarrow \infty} \sigma(t, A)=\infty\right\}\right)=1
$$

for every Borel set A such that $\lambda(A)>0$.

PROOF. (a) In this case the property follows from the fact that

$$
\mathbb{E}\left[\int_{0}^{\infty} \mathbb{1}_{A}\left(Z_{S}\right) d s\right]=\int_{\mathbb{R}} \mathbb{1}_{A}(y) U(y) d y,
$$

where $U(y)=\int_{0}^{\infty} f(s, y) d s$ satisfies the relation $U(y) \leq \frac{c_{2}}{|y|^{1-\alpha}}$ for a suitable constant $c_{2}$ [cf. [17], (16), page 1235], $f(s, y)$ denoting the $\alpha$-stable transition density.

(b) The property depending only on the law of $Z$, we may clearly assume that $Z$ denotes the canonical Markov realization of the $\alpha$-stable Lévy process on the canonical space. Since in the case $Z$ is a recurrent Lévy process in Sato's sense (cf. [15], Theorem 3.12) and has resolvent kernel absolutely continuous with respect to $\lambda$, every Borel set $A$ with $\lambda(A)>0$ is recurrent according to Definition (3.5) in [13], Chapter 10, Section 3 (cf. also [1], Exercise 8, page 40). Measure $\lambda$ being invariant, $Z$ is Harris-recurrent according to Definition (3.8) in [13], Chapter 10, Section 3, so that the property follows from Proposition (3.11) there.

Also in the nonsymmetric case the $0-1$ law in Section 1 of [18] holds [cf. Theorem (1.4) there]. We state it here in a slightly different form, adding some conditions. 
THEOREM $1.2(0-1$ law for $\alpha$-stable motions, $1<\alpha \leq 2)$. Assume that $Z$ has parameter $\alpha$ with $1<\alpha \leq 2$. Let $f$ be a Borel measurable function of the real line into $[0, \infty]$.

Then the following conditions are equivalent:

(a) $P\left(\left\{\int_{0}^{t} f\left(Z_{s}\right) d s<\infty\right.\right.$ for every $\left.\left.t \geq 0\right\}\right)>0$;

(b) $P\left(\left\{\int_{0}^{t} f\left(Z_{s}\right) d s<\infty\right.\right.$ for every $\left.\left.t \geq 0\right\}\right)=1$;

(c) $\int_{K} f(y) d y<\infty$ for every compact subset $K$ of the real line;

(d) for every real-valued random variable $X$ on $(\Omega, \mathcal{F})$ one has

$$
P\left(\left\{\int_{0}^{t} f\left(X+Z_{s}\right) d s<\infty \text { for every } t \geq 0\right\}\right)>0
$$

(e) for every real-valued random variable $X$ on $(\Omega, \mathcal{F})$ one has

$$
P\left(\left\{\int_{0}^{t} f\left(X+Z_{s}\right) d s<\infty \text { for every } t \geq 0\right\}\right)=1
$$

(f) for every $x \in \mathbb{R}, P\left(\left\{\int_{0}^{t} f\left(x+Z_{s}\right) d s<\infty\right.\right.$ for every $\left.\left.t \geq 0\right\}\right)=1$;

(g) for every $x \in \mathbb{R}$ there exists an $\alpha$-stable motion $(\zeta, \mathbb{H}), 1<\alpha \leq 2$, and a finite, strictly positive random variable $\tau$ on a suitable stochastic basis ( $\Xi, \mathcal{H}$, $\mathbb{H}, Q)$ such that

$$
Q\left(\left\{\int_{0}^{\tau} f\left(x+\zeta_{s}\right) d s<\infty\right\}\right)>0 .
$$

The proof uses the following lemma [cf. [18], Lemma (1.6)].

LEMMA 1.3. Let $Z, f$ be respectively a process and a function as in the above theorem. Assume that there exists a real-valued random variable $X$ on $(\Omega, \mathcal{F})$ and a finite and strictly positive random variable $\tau$ such that

$$
P\left(\left\{\int_{0}^{\tau} f\left(X+Z_{s}\right) d s<\infty\right\}\right)>0 .
$$

Then $P(B)>0$, where

$$
B=\{\omega \in \Omega \text { : there exists } \delta(\omega) \in] 0,+\infty\left[\text { with } \int_{-\delta(\omega)}^{\delta(\omega)} f(X(\omega)+y) d y<\infty\right\} .
$$

PROOF. The occupation time density formula (0.2) extends to give, for $P$-a.e. $\omega$

$$
\int_{0}^{t} f\left(X(\omega)+Z_{s}(\omega)\right) d s=\int_{\mathbb{R}} f(X(\omega)+y) L(\omega, t, y) d y \text { for all } t
$$

thus

$$
\int_{0}^{\tau(\omega)} f\left(X(\omega)+Z_{s}(\omega)\right) d s=\int_{\mathbb{R}} f(X(\omega)+y) L(\omega, \tau(\omega), y) d y .
$$


Now the proof is accomplished as that of Lemma (1.6) in [18], using the strict positivity of $\tau$, which implies $P(\{\omega: L(\omega, \tau(\omega), 0)>0\})=1$ and the fact that $L(\omega, \tau(\omega), \cdot)$ is continuous.

REMARK 1.4. In the case when $X$ in the above lemma takes a.s. a constant value $x$, the stated property implies that there exists a real number $\delta>0$ such that

$$
\int_{-\delta}^{\delta} f(x+y) d y<\infty
$$

Proof OF THEOREM 1.2. The proof that condition (a) implies (c) is performed as that of the implication (a) $\Rightarrow$ (c) of Theorem (1.4) in [18], using the above remark.

Suppose now that (c) holds. Fix any $t$ : thanks to relation (1.1), for $P$-a.e. $\omega$ we have

$$
\begin{aligned}
\int_{0}^{t} f\left(X(\omega)+Z_{s}(\omega)\right) d s & =\int_{\mathbb{R}} f(X(\omega)+y) L(\omega, t, y) d y \\
& =\int_{m_{t}(\omega)}^{M_{t}(\omega)} f(X(\omega)+y) L(\omega, t, y) d y
\end{aligned}
$$

where $m_{t}(\omega)$ [resp. $M_{t}(\omega)$ ] denotes the infimum (resp. the supremum) of the path $Z$. $(\omega)$ on the interval $[0, t]$. Then (e) follows using the continuity of $L(\omega, t, \cdot)$.

If $(g)$ is verified, still because of the above remark, for each $x \in \mathbb{R}$ there exists an open neighborhood $U_{x}$ of $x$ such that $\int_{U_{x}} f(x+y) d y<\infty$ and (c) follows by a compactness argument. The proof is completed by simply noting that (e) $\Rightarrow$ (d) $\Rightarrow$ (a), (e) $\Rightarrow$ (b) $\Rightarrow$ (a) and (e) $\Rightarrow$ (f) $\Rightarrow$ (g) .

2. Stochastic differential equations without drift: a necessary condition and the case $1<\alpha \leq 2$. Consider the one-dimensional stochastic differential equation

$$
X_{t}=X_{0}+\int_{10, t]} b\left(X_{s^{-}}\right) d Z_{S}, \quad t \geq 0,
$$

where $X_{0}$ has a given distribution $\eta$ on $\mathbb{R}, b$ is a Borel measurable real function and $Z$ denotes an $\alpha$-stable Lévy process with a given Lévy measure $v$ if $\alpha \neq 2$; of course, when $\alpha=1$, the drift coefficient $\gamma$ also is given $(\gamma=0$ if $Z$ is a Cauchy process).

In the following, when $\alpha \neq 2$, we always make the assumption that either $Z$ is symmetric or $b$ is nonnegative; however, for the sake of brevity, we omit it in the statements.

Here we investigate weak solutions of the above equation which we simply call solutions. Also we sometimes employ the term "global" to mean solutions defined for all $t \geq 0$, to be distinguished from "local" solutions introduced below. 
Everywhere in the following $f(t, y)$ denotes the $\alpha$-stable transition density $(0<\alpha \leq 2)$.

As in [18] we call trivial a solution $X$ such that, a.s., $X_{t}=X_{0}$ for all $t$.

We start with the following necessary condition of existence of nontrivial solutions [cf. [18], Proposition (2.30)]. [It is perhaps worth noticing that the necessary existence conditions below hold without any restriction on the sign of $b$ (cf. Propositions 2.1 and 3.12). When $Z$ is nonsymmetric the restriction on $b$ is used only for the sufficient conditions of existence.]

Proposition 2.1. Let $\alpha, 0<\alpha \leq 2$, be fixed and let $Z, X$ respectively denote an $\alpha$-stable Lévy motion and a process defined on a basis $\mathbb{B}=(\Omega, \mathcal{F}, \mathbb{F}, P)$ and solving the above equation. Assume that $X$ is nontrivial, with initial law $\eta$. Then there exists a real number $t>0$ such that

$$
P\left(\left\{\int_{0}^{t} \frac{d s}{\left|b\left(X_{0}+Z_{s}\right)\right|^{\alpha}}<\infty\right\}\right)>0 .
$$

Moreover, when $1<\alpha \leq 2$ and $\eta$ is Dirac measure $\varepsilon_{x}, x \in \mathbb{R}$, there exists a real number $\varepsilon>0$ such that

$$
\int_{|y| \leq \varepsilon} \frac{d y}{|b(x+y)|^{\alpha}}<+\infty
$$

Proof. Consider process $\tau_{t}=\int_{0}^{t}\left|b\left(X_{s^{-}}\right)\right|^{\alpha} d s$ which is a.s. finite (see [8], Theorem 3.1): because of the random time change representation of stable integrals (see [8], Theorem 4.1), the solution can be represented for all $t$ as follows:

$$
X_{t}=X_{0}+\tilde{Z}_{\tau_{t}},
$$

where $\tilde{Z}$ is an $\alpha$-stable Lévy motion with the same law as $Z$, defined in general on an extension of the original probability space $(\Omega, \mathcal{F}, P)$, which we still denote by the same symbol. If $C$ is the right inverse of $\tau$ defined for all $t$ as

$$
C_{t}=\inf \left\{s \geq 0: \tau_{s}>t\right\},
$$

taking the above relation into account, by time change in the integral we have, a.s.,

$$
\begin{aligned}
C_{t} & \geq \int_{0}^{C_{t}} \mathbb{1}_{\left\{b\left(X_{s}\right) \neq 0\right\}} d s=\int_{0}^{C_{t}}\left|b\left(X_{s}\right)\right|^{-\alpha}\left|b\left(X_{s}\right)\right|^{\alpha} d s=\int_{0}^{C_{t}}\left|b\left(X_{s}\right)\right|^{-\alpha} d \tau_{s} \\
& =\int_{0}^{t \wedge \tau_{\infty}}\left|b\left(X_{0}+\tilde{Z}_{s}\right)\right|^{-\alpha} d s \quad \text { for all } t \geq 0 \text { [cf. [3], Lemma (1.6)]. }
\end{aligned}
$$

Because of the nontriviality of $X$, one has $P\left(\left\{\tau_{\infty}>0\right\}\right)>0$ so that there exists $t>0$ with $P\left(\left\{\tau_{\infty}>t\right\}\right)>0$. But $C_{t}$ is finite on the set $\left\{t<\tau_{\infty}\right\}$ and from the above relation it follows that, for that $t$,

$$
P\left(\left\{\int_{0}^{t} \frac{d s}{\left|b\left(X_{0}+\tilde{Z}_{s}\right)\right|^{\alpha}}<\infty\right\}\right)>0
$$


The conclusion follows because of the measurability of the function $\gamma:(\mathbb{D}, \mathbb{D}) \rightarrow$ $[0, \infty], \gamma(\sigma)=\int_{0}^{t} \frac{d s}{\left|b\left(\sigma_{s}\right)\right|^{\alpha}}$, process $X_{0}+Z$ having the same law as $X_{0}+\tilde{Z}$.

The second statement follows from Remark 1.4.

In the case of (2.1) driven by $Z$ with $1<\alpha \leq 2$, here we completely characterize weak existence and uniqueness. The results depend on integrability properties of $|b|^{-\alpha}$.

Let us introduce the sets

$$
\begin{aligned}
& N=\{x \in \mathbb{R}: b(x)=0\}, \\
& I=\left\{x \in \mathbb{R}: \int_{-\varepsilon}^{+\varepsilon} \frac{d y}{|b(x+y)|^{\alpha}}=\infty \text { for any } \varepsilon>0\right\}
\end{aligned}
$$

[we set $|b(y)|^{-\alpha}=+\infty$ if $b(y)=0$ ].

THEOREM 2.2. Given $\alpha, 1<\alpha \leq 2$, consider (2.1) driven by $Z$ with parameter $\alpha$. For every law $\eta$ on $\mathbb{R}$, the equation has a solution with initial distribution $\eta$ if and only if $I \subset N$.

The property reducing in the continuous case to Theorem 1 in [4], we consider the case $1<\alpha<2$ and first prove a basic lemma.

Consider a stochastic basis $\mathbb{B}$ carrying a strictly $\alpha$-stable Lévy process $Z$ with arbitrary initial law.

Let the increasing process $C$ be defined by the formula

$$
C_{t}=\int_{0}^{t} \frac{d s}{\left|b\left(Z_{s}\right)\right|^{\alpha}}, \quad t \geq 0 .
$$

Using relation (1.1) and the fact that a.s. $\lim _{t \rightarrow \infty} L(t, y)=\infty$ for all $y$, where $L$ denotes the local time of $Z$ (cf. [16], Theorem 1), we have, a.s.,

$$
\lim _{t \rightarrow \infty} C_{t}=\infty \text {. }
$$

Consider the first entrance time $D$ into the closed set $I$ of (2.3) for process $Z$ :

$$
D=\inf \left\{t \geq 0: Z_{t} \in I\right\} .
$$

LEMMA 2.3. We have a.s.:

(a) $C_{t}<\infty$ for all $t<D$;

(b) $C_{D^{+}}=\infty$.

(Of course, when $D=\infty, C_{D^{+}}=C_{D}$.) 
Proof. Denote by $\delta(x, I)=\inf \{|x-y|: y \in I\}$ the distance of $x \in \mathbb{R}$ from $I$. For the sets $I_{n}=\left\{x \in \mathbb{R}: \delta(x, I)<\frac{1}{n}\right\}, n \geq 1$, define the stopping times

$$
D_{n}=\inf \left\{t \geq 0: Z_{t} \in I_{n}\right\}
$$

Sequence $\left(D_{n}\right)_{n \geq 1}$ is increasing with $n$ and because of the quasi-left-continuity of $Z$, we have a.s. (see [1], Corollary 8, page 22)

$$
\lim _{n \rightarrow \infty} D_{n}=D \text {. }
$$

Now if we set, for each $n, b_{n}=b \cdot \mathbb{1}_{I_{n}^{c}}+1 \cdot \mathbb{1}_{I_{n}},\left|b_{n}\right|^{-\alpha}$ is locally integrable and from Theorem 1.2(e) we have, a.s.,

$$
\int_{0}^{t} \frac{d s}{\left|b_{n}\left(Z_{s}\right)\right|^{\alpha}}=\int_{0}^{t} \frac{d s}{\left|b\left(Z_{s}\right)\right|^{\alpha}}<\infty \quad \text { for all } t<D_{n} .
$$

Assertion (a) follows from the above limit relation.

To prove (b), because of (2.5) we may clearly assume $I \neq \varnothing$, so that $D$ is a.s. finite and, for every $t>0$,

$$
\int_{0}^{D+t} \frac{d s}{\left|b\left(Z_{s}\right)\right|^{\alpha}} \geq \int_{0}^{t} \frac{d s}{\left|b\left(Z_{D}+Z_{s}^{*}\right)\right|^{\alpha}}
$$

where $Z_{s}^{*}=Z_{D+s}-Z_{D}$ is an $\alpha$-stable motion starting from 0 .

Since $I$ is closed, $Z_{D} \in I$ a.s. and the right-hand-side integral is a.s. infinite, thanks to Lemma 1.3. Thus $C_{D^{+}}=\infty$ a.s.

Proof of TheOrem 2.2. Necessity of condition $I \subset N$ is a consequence of Proposition 2.1. Neglecting the trivial case of a null coefficient, suppose that $X$ is a solution starting from $x$ with $b(x) \neq 0$. Such a solution turns out to be nontrivial and that proposition implies $x \notin I$.

To prove sufficiency, the parameter $\alpha, 1<\alpha<2$, and the law $\eta$ being fixed, let $Z$ denote an $\alpha$-stable Lévy process $Z$ with initial law $\eta$ and Lévy measure $v$, defined on a stochastic basis $(\Omega, \mathcal{F}, \mathbb{F}, P)$. Define process $C$ by (2.4): then $C_{0}=0, C_{t}>0$ for all $t>0$ and thanks to the above lemma, a.s. $C$ is continuous and strictly increasing on the interval $\left[0, D\right.$ [ and for $t>D$ one has $C_{t}=\infty$. Note that $C_{D}$ may be finite when $D$ is finite, in which case $C$ jumps from $C_{D}$ to $\infty$ at time $D$.

Now introduce the right inverse of $C$,

$$
\tau_{t}=\inf \left\{s \geq 0: C_{s}>t\right\}, \quad t \geq 0 .
$$

Process $\tau$ satisfies $\tau_{0}=0$, is increasing, is a.s. finite for all $t$ thanks to (2.5) and

$$
\tau_{\infty}=\lim _{t \rightarrow \infty} \tau_{t}=\inf \left\{s: C_{s}=\infty\right\}
$$

From the above lemma we have, a.s.,

$$
D=\tau_{\infty}
$$


Clearly a.s. $\tau$ is everywhere continuous and strictly increasing on $\left[0, C_{D}[\right.$ and if $C_{D}<\infty$, we have $\tau_{t}=\tau_{\infty}=D$ for all $t \geq C_{D}$. Also note that, a.s.,

$$
C_{D}=C_{\tau_{\infty}}=\inf \left\{t: \tau_{t}=\tau_{\infty}\right\} \quad \text { and } \quad C_{s^{+}}=\inf \left\{t: \tau_{t}>s\right\}, \quad s \geq 0 .
$$

Clearly $\tau=\left(\tau_{t}\right)_{t \geq 0}$ is a change of time. Let us define the time-changed process $(X, \mathbb{H})$ as follows:

$$
X_{t}=Z_{\tau_{t}}, \quad \mathbb{H}=\left(\mathscr{H}_{t}\right)_{t \geq 0} \quad \text { where } \mathscr{H}_{t}=\mathcal{F}_{\tau_{t}} .
$$

We will show that the process $X$ is a solution of (2.1) on an extension of $\mathbb{B}=(\Omega, \mathcal{F}, \mathbb{H}, P)$.

Since the basis $(\Omega, \mathcal{F}, \mathbb{F}, P)$ satisfies the usual assumptions, the same holds for $\mathbb{B}$. Let $\mu$ denote the jump-measure of $(Z, \mathbb{F})$, that is, the integer-valued random measure

$$
\mu(d t, d x)=\sum_{s>0} \mathbb{1}_{\left\{\Delta Z_{s} \neq 0\right\}} \varepsilon_{\left(s, \Delta Z_{s}\right)}(d t, d x),
$$

where $\varepsilon_{(s, x)}$ denotes the Dirac measure concentrated at point $(s, x)$.

Define the time-changed random measure $\tilde{\mu}=\tau \mu$ as follows: for every $\omega$,

$$
\begin{aligned}
\tilde{\mu}(\omega ; \cdot)=(\tau \mu)(\omega ; \cdot)=\mu(\omega ; \cdot) \circ(\tilde{C}(\omega))^{-1} \\
\text { where } \tilde{C}(\omega)(t, x)=\left(C_{t}(\omega), x\right) .
\end{aligned}
$$

Because of Theorem (10.27)(b), (e) in [6], $\tilde{\mu}$ is the jump-measure of the process $(X, \mathbb{H})$ and the $\mathbb{H}$-dual predictable projection $\tilde{\pi}$ of $\tilde{\mu}=\tau \mu$ is given by $\tau \pi$; that is, for every $\omega$,

$$
\tilde{\pi}(\omega ; \cdot)=(\tau \pi)(\omega ; \cdot)=\pi(\omega ; \cdot) \circ(\tilde{C}(\omega))^{-1},
$$

where $\pi(d t, d x)=d t \otimes v(d x)$ denotes the $\mathbb{F}$-compensator of $\mu$ and $v$ is the Lévy measure of $Z$.

As a next step we compute the explicit form of $\tau$ of (2.7) showing that, a.s.,

$$
\tau_{t}=\int_{0}^{t}\left|b\left(X_{s}\right)\right|^{\alpha} d s \quad \text { for all } t \geq 0 .
$$

We fix an $\omega$ such that $\tau$. $(\omega)$ is everywhere continuous, $C_{\infty}(\omega)=\infty, \tau_{\infty}(\omega)=$ $D(\omega)$ but we omit it in the notation. If $\tau_{\infty}=0=D$, the path $\tau$. is constantly zero so that $X$ constantly assumes the value $X_{0}=Z_{0} \in I$ : the assumption entails $b\left(X_{s}\right)=b\left(Z_{0}\right)=0$ for all $s$ and the above relation holds with both sides 0 .

If $\tau_{\infty}>0$, consider $t$ with $0 \leq t<C_{\tau_{\infty}}$, that is, $\tau_{t}<\tau_{\infty}$ : then $C_{\tau_{t}}<\infty$; thus $b\left(Z_{s}\right)$ is not 0 for $\lambda$-a.e. $s \in\left[0, \tau_{t}\right]$ and by time change in the integral we have

$$
\tau_{t}=\int_{0}^{\tau_{t}}\left|b\left(Z_{s}\right)\right|^{\alpha} d C_{s}=\int_{0}^{t}\left|b\left(X_{s}\right)\right|^{\alpha} d s .
$$


So, when $C_{\tau_{\infty}}=\infty$, we are done. Otherwise, when $C_{\tau_{\infty}}<\infty$, we have $\tau_{\infty}<\infty$ and, thanks to the continuity of $\tau$, letting $t \uparrow C_{\tau_{\infty}}$ in the last relation we have

$$
\tau_{\infty}=\int_{0}^{C_{\tau_{\infty}}}\left|b\left(X_{s}\right)\right|^{\alpha} d s,
$$

so that (2.12) holds for all $t, 0 \leq t \leq C_{\tau_{\infty}}=C_{D}$. But $X_{C_{\tau_{\infty}}}=Z_{\tau_{\infty}}=Z_{D} \in I$ and the assumption implies $b\left(X_{C_{\tau_{\infty}}}\right)=0$. For all $t>C_{\tau_{\infty}}$ we have $\tau_{t}=\tau_{\infty}$ so that $b\left(X_{t}\right)=b\left(Z_{\tau_{t}}\right)=b\left(Z_{\tau_{\infty}}\right)=0$ and, for those $t,(2.12)$ holds with both sides equal to $\tau_{\infty}$. The validity of (2.12) is proved.

Since (2.11) can be written $\tilde{\pi}(\omega ; d t, d x)=d \tau_{t}(\omega) \otimes v(d x)$, from (2.12) we conclude that $\tilde{\pi}$ satisfies the following relation, up to $P$-equivalence:

$$
\tilde{\pi}(\omega ; d t, d x)=\left|b\left(X_{t^{-}}(\omega)\right)\right|^{\alpha} d t \otimes v(d x) .
$$

However, $Z$ is a purely discontinuous $\mathbb{F}$-martingale and, thanks to Theorem (10.16) of [6], process $(X, \mathbb{H})$ is a purely discontinuous local martingale on $\mathbb{B}$. Because of the last relation, in the case when $b$ is nonnegative (resp. when $Z$ is symmetric) we can employ (a) [resp. (c)] of Theorem 2 in [19] to conclude that the following representation formula holds:

$$
X_{t}-Z_{0}=\int_{] 0, t]} b\left(X_{s^{-}}\right) d Z_{s}^{*}, \quad t \geq 0,
$$

where $Z^{*}$ is an $\alpha$-stable Lévy process with the same Lévy measure as $Z$, defined in general on an extension of $\mathbb{B}$. Thanks to the definition of $X$ the proof is over.

REMARK 2.4. Let $X$ be the solution constructed in the sufficiency part of the above proof, such that a.s. $X_{0}=Z_{0}=x \in I^{c}$. Then, the notation being as there, a.s. we have $D>0$; thus $C_{D}>0$, and $\tau$. being strictly increasing on the interval $\left[0, C_{D}\right.$ [, a.s. the path $X .=Z_{\tau}$. is not identically constant (so, in particular, the solution is nontrivial)

On the other hand, if $x \in N, X_{t}=x$ identically is a trivial solution of (2.1). The conclusion is that there can be no uniqueness in law if $N$ is strictly larger than $I$.

EXAMPLE 2.5. Still with reference to the sufficiency part of the above proof, one can easily construct examples where $D$ and $C_{D}$ are a.s. finite. So, in the case of (2.1) driven by $Z$ with no positive jumps (i.e., with Lévy measure $v$ vanishing on $] 0,+\infty[)$, define $b, b(y)=1 \cdot \mathbb{1}_{]-\infty, 1[}(y)+(y-1) \cdot \mathbb{1}_{[1,+\infty[}(y)$ and let $\eta$ be Dirac measure $\varepsilon_{0}$. Then $I=\{1\}=N$. Let $D$ be defined starting from a $Z$ as above, $Z_{0}=0$ a.s. Then $0<D<\infty$ a.s. and since $Z$ does not jump across 1 before time $D$ a.s. (cf. [1], Proposition 8, page 226), we have $C_{t}=t$ for all $t<D$; thus $C_{D}=D$ a.s.

THEOREM 2.6. Assumptions are the same as in the preceding theorem. For every initial distribution, (2.1) has a solution unique in law if and only if $I=N$. 
Proof. Necessity. Condition $I \subset N$ is necessary for existence and, adding uniqueness, the above remark shows that $I=N$.

Sufficiency. We show that condition $N \subset I$ is sufficient for uniqueness.

Parameter $\alpha, 1<\alpha<2$, being fixed, let $X$ denote a solution of (2.1) with initial distribution $\eta$. As in the proof of Proposition 2.1, $X$ can be represented as follows:

$$
X_{t}=Z_{\tau_{t}}, \quad t \geq 0,
$$

where $\tau_{t}=\int_{0}^{t}\left|b\left(X_{s^{-}}\right)\right|^{\alpha} d s$ and $Z$ is an $\alpha$-stable Lévy motion with initial law $\eta$, defined in general on an extension of the original probability space.

Now we show that the right inverse of $\tau, C_{t}=\inf \left\{s: \tau_{s}>t\right\}$, satisfies a.s. the following relation:

$$
C_{t}=\int_{0}^{t^{+}} \frac{d s}{\left|b\left(Z_{s}\right)\right|^{\alpha}} \quad \text { for all } t \geq 0
$$

the proof is similar to the case $\alpha=2$ and we sketch it for the sake of completeness. It is based on Lemma 2.3 and on the analogous one of relation (2.2): a.s.,

$$
\begin{aligned}
C_{t} & \geq \int_{0}^{C_{t}} \mathbb{1}_{\left\{b\left(X_{s}\right) \neq 0\right\}} d s=\int_{0}^{t \wedge \tau_{\infty}} \frac{d s}{\mid b\left(X_{C_{s}}\right)^{\alpha}} \\
& =\int_{0}^{t \wedge \tau_{\infty}} \frac{d s}{\left|b\left(Z_{s}\right)\right|^{\alpha}} \quad \text { for all } t .
\end{aligned}
$$

Now we introduce the stopping time

$$
T=\inf \left\{t \geq 0: X_{t} \in I\right\},
$$

while $D$ denotes the first entrance time into $I$ of (2.3) for process $Z$.

If $T>0$, for every $t, 0 \leq t<T$, we have $X_{t} \notin I$ so that $b\left(X_{t}\right) \neq 0$ thanks to the assumption: it follows that process $\tau$ is strictly increasing on the interval $[0, T[$.

Since relation $b\left(X_{S}\right) \neq 0$ for all $s \geq 0$ holds on $\{T=\infty\}$, because of (2.19) on this set we have, a.s.,

$$
C_{t}=\int_{0}^{t \wedge \tau_{\infty}} \frac{d s}{\left|b\left(Z_{S}\right)\right|^{\alpha}} \text { for all } t
$$

thus, when $t \geq \tau_{\infty}, C_{t}=\infty$ and (2.18) holds with both sides equal to $\infty$; when $t<\tau_{\infty}$ we have $C_{t}=\int_{0}^{t} \frac{d s}{\left|b\left(Z_{s}\right)\right|^{\alpha}}$, which is relation (2.18), since $C$ is continuous on $\left[0, \tau_{\infty}[, \tau\right.$ being now strictly increasing on $[0, \infty[$. So (2.18) holds a.s. on $\{T=\infty\}$.

Moreover, by a simple verification based on the fact that $\tau$ is strictly increasing on $\left[0, T\left[\right.\right.$ and everywhere continuous, we see that a.s. $\tau_{T}=D$ on the set $\{T<\infty\}$. Also, thanks to (2.19), note that the set $\left\{D<\tau_{\infty}\right\}$ is a.s. included in $\left\{\int_{0}^{D^{+}} \frac{d s}{\left.b\left(Z_{s}\right)\right|^{\alpha}}<\infty\right\}$ so that Lemma 2.3 entails $D \geq \tau_{\infty}$, a.s. We deduce that the following holds:

$$
\tau_{T}=\tau_{\infty}=D \quad \text { a.s. on the set }\{T<\infty\}
$$


So, still taking Lemma 2.3 into account, (2.18) holds a.s. on $\{T=0\}$ with both sides equal to $\infty$ for all $t$.

Now, process $C$ being continuous on $\left[0, \tau_{T}[\right.$, on the set $\{0<T<\infty\}$ relation (2.18) is a.s. equivalent to

$$
C_{t}=\int_{0}^{t} \frac{d s}{\left|b\left(Z_{s}\right)\right|^{\alpha}} \quad \text { for all } t<\tau_{T}=\tau_{\infty}<\infty
$$

since (2.18) is automatically satisfied for all $t \geq \tau_{\infty}=D$ because of Lemma 2.3 and the very definition of $C$. But last relation is still derived from (2.19) thanks to the fact that $t<\tau_{T}$ implies $b\left(X_{s}\right) \neq 0$ for all $s, 0 \leq s \leq C_{t}<T=C_{\left(\tau_{T}\right)^{-}}$.

So (2.18) is shown. It follows that $C$ and $\tau$ are linked as in the sufficiency part of the proof of Theorem 2.2, that is,

$$
\tau_{s}=\inf \left\{t: \int_{0}^{t^{+}} \frac{d s}{\left|b\left(Z_{s}\right)\right|^{\alpha}}>s\right\}=\inf \left\{t: \int_{0}^{t} \frac{d s}{\left|b\left(Z_{s}\right)\right|^{\alpha}}>s\right\} \text { for all } s, \text { a.s., }
$$

and from (2.17) we conclude that $X$ is a measurable function of the process $Z$ [in the sense of functions defined on the Skorokhod space $(\mathbb{D}, \mathscr{D})$ ]. Thus the law of $X$ is entirely determined by that of the process $Z$, which in turn depends only on $\eta$.

3. Nontrivial solutions and local solutions. To study (2.1) driven by the $Z$ 's with parameter $\alpha, 0<\alpha \leq 1$, we recall the following definition [see [18], Definition (2.4)]:

DEFINITION 3.1. Let $x$ be a real number. We say that the coefficient $b$ in (2.1) satisfies condition $(\mathrm{H})$ with respect to $x$ [for short, $(\mathrm{H})(x)]$ if

$$
\int_{0}^{t} d s\left(\int_{|y|<L} \frac{1}{|b(x+y)|^{\alpha}} f(s, y) d y\right)<\infty \text { for all } t>0, L>0
$$

( $f$ denoting the $\alpha$-stable transition density, $0<\alpha \leq 2$ ). [We set $|b(x)|^{-\alpha}=+\infty$ if $b(x)=0$.

Here we also introduce the following "local" version of $(\mathrm{H})$ :

DEFINITION 3.2. Let $x$ be a real number. We say that the coefficient $b$ in (2.1) satisfies condition $(\mathrm{LH})$ with respect to $x$ [for short $(\mathrm{LH})(x)$ ] if there exists a real number $\varepsilon>0$ such that

$$
\int_{0}^{\varepsilon} d s\left(\int_{|y| \leq \varepsilon} \frac{1}{|b(x+y)|^{\alpha}} f(s, y) d y\right)<\infty .
$$

A first application of condition $(\mathrm{H})(x)$ leads to the following theorem which improves Theorem (2.5) of [18] including the case of equations driven by a 1-stable Lévy process and removing the symmetry assumption in [18]. With reference to this, recall the assumption fixed at the beginning of Section 2. 
THEOREM 3.3. Consider (2.1). Let $x$ be such that function $b$ satisfies condition $(\mathrm{H})(x)$ :

(a) Let $1 \leq \alpha \leq 2$. Under the stated assumptions, there exists a nontrivial solution starting from $x$ (i.e., such that $X_{0}=x$ a.s.).

(b) Let $0<\alpha<1$. In addition to the above assumptions, suppose that there exists a real number $U>0$ such that $\lambda\left(B_{U}\right)<\infty$, where

$$
B_{U}=\{y \in \mathbb{R}:|b(y)|>U\} .
$$

Then there exists a nontrivial solution starting from $x$.

The proof uses results from [19] and Proposition 1.1. Begin by considering an $\alpha$-stable Lévy process $(Z, \mathbb{F})$ defined on some stochastic basis $\mathbb{B}=(\Omega, \mathcal{F}, \mathbb{F}, P)$ and starting from $x$. As in Section 2, a solution of (2.1) will be constructed by random time change from $Z$.

For $0<\alpha \leq 2$ define the process $(C, \mathbb{F})$ by $(2.4)$.

Lemma 3.4. Process $C$ satisfies the following properties:

(a) If $b$ in (2.1) satisfies condition $(\mathrm{H})$ with respect to $x$, then

$$
P\left(\left\{C_{t}<\infty \text { for every } t\right\}\right)=1 .
$$

(b) When $1 \leq \alpha \leq 2$, for an arbitrary $b$ we have $\lim _{t \rightarrow \infty} C_{t}=C_{\infty}=\infty$, a.s. When $0<\alpha<1$ the same holds for a coefficient $b$ satisfying the last assumption in (b) of the above theorem.

PROOF. Property (a) follows from condition $(\mathrm{H})(x)$ as in the proof of Lemma (2.7) in [18].

Property (b), in the case $1<\alpha \leq 2$, has already been noted [cf. (2.5)]. Moreover, for $b$ arbitrary, there exists $\varepsilon>0$ such that $\lambda\left(B_{\varepsilon}\right)>0$, where $B_{\varepsilon}=$ $\left\{y \in \mathbb{R}: \frac{1}{|b(y)|} \geq \varepsilon\right\}$. Thus, in the case $\alpha=1$, the property follows from the fact that

$$
C_{t}(\omega) \geq \int_{0}^{t} \frac{1}{\left|b\left(Z_{s}\right)\right|} \mathbb{1}_{\left\{Z_{s} \in B_{\varepsilon}\right\}}(s) d s \geq \varepsilon \int_{0}^{t} \mathbb{1}_{\left\{Z_{s} \in B_{\varepsilon}\right\}}(s) d s,
$$

employing Proposition 1.1(b) for the occupation time measure of process $Z-x$. Also the case $0<\alpha<1$ is a consequence of Proposition 1.1(a) (cf. the proof of the above-quoted lemma).

ProOF OF THEOREM 3.3. (a). First we consider the case of (2.1) driven by a 1-stable Lévy process with drift $\gamma \cdot t$ and define $C$ as in (2.4) starting from such a process $Z$ [on $\mathbb{B}=(\Omega, \mathcal{F}, \mathbb{F}, P)$ with $Z_{0}=x$ ]. Recall that in the case $\gamma \neq 0$ the coefficient $b$ is assumed to be nonnegative. 
If $\tau$ denotes the right inverse of $C, \tau_{t}=\inf \left\{s: C_{s}>t\right\}$, thanks to the above lemma, process $\tau$ is a.s. finite, continuous, strictly increasing and satisfies relation

$$
\lim _{t \rightarrow \infty} \tau_{t}=\tau_{\infty}=\infty
$$

Also, because of Lemma 3.4(a) a.s. we have $\left|b\left(Z_{s}\right)\right|>0$ for $\lambda$-a.e. $s$ so that, by change of variable,

$$
\tau_{t}=\int_{0}^{t}\left|b\left(Z_{\tau_{s}}\right)\right| d s \text { for all } t .
$$

Now the proof follows the same lines as the sufficiency part in Theorem 2.2: by the change of time $\tau=\left(\tau_{t}\right)_{t \geq 0}$ one defines the process $(X, \mathbb{H})$ by (2.9) and, as there, the jump-measure $\tilde{\mu}$ of $(X, \mathbb{H})$ is seen to satisfy relation $(2.10)$ and to have $\mathbb{H}$-compensator $\tilde{\pi}$ satisfying the following relation:

$$
\tilde{\pi}(d t, d y)=\left|b\left(X_{t^{-}}\right)\right| d t \otimes v(d y),
$$

where $v(d y)$ is Lévy measure of $Z$. Recall now that the process $Z$ can be represented in terms of its jump-measure $\mu$ as follows, for every $t$ :

$$
Z_{t}=x+\int_{00, t]} \int_{|y| \leq 1} y(\mu-\pi)(d s, d y)+\int_{0, t]} \int_{|y|>1} y \mu(d s, d y)+\gamma t,
$$

$\pi(d t, d y)=d t \otimes v(d y)$ being the $\mathbb{F}$-compensator of $\mu$. So, thanks to Theorems (10.27)(a) and (10.28)(a) in [6], we have, for all $t$,

$$
X_{t}=x+\int_{] 0, t]} \int_{|y| \leq 1} y(\tilde{\mu}-\tilde{\pi})(d s, d y)+\int_{] 0, t]} \int_{|y|>1} y \tilde{\mu}(d s, d y)+\gamma \tau_{t},
$$

so that

$X_{t}=x+\int_{] 0, t]} \int_{|y| \leq 1} y(\tilde{\mu}-\tilde{\pi})(d s, d y)+\int_{] 0, t]} \int_{|y|>1} y \tilde{\mu}(d s, d y)+\gamma \int_{0}^{t}\left|b\left(X_{s}\right)\right| d s$,

thanks to (3.2) and the very definition of $X$.

Such a decomposition of $X$ and property (3.3) permit us to employ some results from [19]. So in the case when $\gamma \neq 0$ (resp. when $\gamma=0$, i.e., in the case of a driving Cauchy process) we use Theorem 1 (b) (resp. the Corollary) there to conclude that the following representation holds:

$$
X_{t}-x=\int_{] 0, t]} b\left(X_{s^{-}}\right) d Z_{s}^{*}, \quad t \geq 0,
$$

where $Z^{*}$ is a 1 -stable Lévy process with drift $\gamma t$ and Lévy measure $v$ (resp. a Cauchy process with Lévy measure $v$ ) defined in general on an extension of $(\Omega, \mathcal{F}, \mathbb{H}, P)$. Thus $X$ solves $(2.1)$ on the extension and thanks to (3.1) this solution is obviously nontrivial.

To complete the proof of (a), it suffices to consider the case $1<\alpha<2$ [for $\alpha=2$ cf. [4], Theorem (2.2)]. As above, starting from an $\alpha$-stable Lévy process $Z$ with 
$Z_{0}=x$, one defines $C$ by (2.4) and combining Lemmas 3.4(a) and 2.3 one sees that $D=\infty=\tau_{\infty}$ a.s., $D$ (resp. $\tau$ ) being defined by (2.6) [resp. (2.7)]. It follows [cf. (2.13)], a.s.,

$$
\tau_{t}=\int_{0}^{t}\left|b\left(Z_{\tau_{s}}\right)\right|^{\alpha} d s \text { for all } t,
$$

and a solution is constructed from $Z$ as in the proof of Theorem 2.2. Such solution is nontrivial as in the case $I=\emptyset$ (cf. Remark 2.4).

(b) The proof goes similarly and in the case when $b$ is nonnegative (resp. when $Z$ is symmetric) one employs (b) [resp. (c)] of Theorem 2 in [19] to obtain the representation formula corresponding to (3.4). We omit the details.

In the above proof, process $C$ never explodes at a finite time due to the fact that the coefficient $b$ satisfies condition $(\mathrm{H})(x)$. When $1<\alpha \leq 2$ this is a restriction with respect to the setting in the preceding section (where $C$ may reach infinity at a finite time) and Theorem 3.15 below will clarify the meaning of condition $(\mathrm{H})(x)$ in the case. However, Theorem 3.3 is preliminary to the proof of the general "local" existence result expressed in Theorem 3.10 below.

REMARK 3.5. In Theorem 3.3 the $\alpha$-stable motion $Z^{*}$ solving the equation [i.e., permitting representation (3.4) in the different cases] can be defined on the same basis where $X$ is defined, which has always been denoted $(\Omega, \mathcal{F}, \mathbb{H}, P)$.

Indeed, using the same notation as in the proofs, set $N_{\omega}=\left\{s \in \mathbb{R}_{+}: b\left(X_{s}(\omega)\right)=\right.$ $\left.b\left(Z_{\tau_{s}}(\omega)\right)=0\right\}$. Then, for $P$-almost every $\omega, \lambda\left(N_{\omega}\right)=0$.

To see this, set $I_{\omega}=\left\{s \in \mathbb{R}_{+}: b\left(Z_{s}(\omega)\right)=0\right\}$. Since a.s. $C_{\infty}=\infty$, because of Theorem 44 in [2], page 92, we have

$$
\lambda\left(N_{\omega}\right)=\int_{0}^{C_{\infty}} \mathbb{1}_{N_{\omega}}(s) d s=\int_{0}^{\infty} \mathbb{1}_{N_{\omega}}(C(t)) d C(t) .
$$

However, $N_{\omega}=C\left(I_{\omega}\right)$ and $\mathbb{1}_{N_{\omega}}(C(t))=\mathbb{1}_{C\left(I_{\omega}\right)}(C(t))=\mathbb{1}_{I_{\omega}}(t)$ for all $t$, because $C$ is strictly increasing. Thus, for $P$-almost every $\omega, \lambda\left(N_{\omega}\right)=$ $\int_{0}^{\infty} \mathbb{1}_{I_{\omega}}(t) d C(t)=0$ since $\lambda\left(I_{\omega}\right)=0$ thanks to the finiteness of $C$ for all $t$ [cf. Lemma 3.4(a)].

Now the property follows applying Proposition 2 of [19] to the process $H_{S}(\omega)=$ $b\left(X_{s^{-}}(\omega)\right)$.

REMARK 3.6. Let $\mathbb{B}=(\Omega, \mathcal{F}, \mathbb{F}, P)$ denote a stochastic basis carrying an $\alpha$-stable Lévy process $(Z, \mathbb{F})$ with $Z_{0}=0$ and an independent random variable $V$ which has a given law $\eta$ and is $\mathcal{F}_{0}$-measurable. Assume that for any $x \in \mathbb{R}$ process $C_{t}^{x}=\int_{0}^{t} \frac{d s}{\left|b\left(x+Z_{s}\right)\right|^{\alpha}}$ satisfies the following relations:

(a) $P\left(\left\{C_{t}^{x}<\infty\right.\right.$ for every $\left.\left.t\right\}\right)=1$;

(b) $P\left(\left\{\lim _{t \rightarrow \infty} C_{t}^{x}=\infty\right\}\right)=1$.

Then the corresponding relations are met by the process $C_{t}^{V}=\int_{0}^{t} \frac{1}{\left|b\left(V+Z_{s}\right)\right|^{\alpha}} d s$. 
This is seen in a standard way using the measurability of the function $\Gamma(\sigma)=$ $\int_{0}^{t} \frac{1}{|b(\sigma(s))|^{\alpha}} d s$ from $(\mathbb{D}, \mathscr{D})$ into $[0, \infty]$ and the relation between the law of process $V+Z$., stable with initial law $\eta$, and the laws of the processes $x+Z, x \in \mathbb{R}$. Thus, repeating the proof of Theorem 3.3 starting from the process $C^{V}$, we conclude that for (2.1) there exists a nontrivial solution with initial law $\eta$, where $\eta$ is arbitrary, under conditions assuring the validity of (a), (b) above for all $x$.

The following property relates condition $(\mathrm{H})(x)$ (Definition 3.1) to integrability properties of the coefficient $b$ alone and improves Proposition (2.29) in [18] adding the case $\alpha=1$ and removing the symmetry assumption there when $\alpha \neq 2$. It is proven as in that proposition simply noting that relations (2.27) and (2.28) in [18] hold also in the nonsymmetric case for all $\alpha$.

PROPOSITION 3.7. (a) In the case $1<\alpha \leq 2$, assume that the following holds: $|b|^{-\alpha}$ is locally integrable (with respect to $\lambda$ ).

(b) In the case $0<\alpha \leq 1$ assume that there exists a real number $\delta>1$ such that the following holds: $|b|^{-\delta}$ is locally integrable.

Then in both cases coefficient $b$ in (2.1) satisfies condition (H) (Definition 3.1) with respect to any $x$.

Combining the last proposition with Theorem 3.3 and Remark 3.6, we immediately obtain sufficient conditions of existence for (2.1) based only on $b$. In particular, we obtain the following.

THEOREM 3.8. Consider (2.1) driven by a Cauchy process [resp. (2.1) with a nonnegative $b$, driven by a 1 -stable Lévy process with nonvanishing drift]. Assume that there exists a real number $\delta>1$ such that $|b|^{-\delta}$ is locally integrable. Then for any law $\eta$ there exists a nontrivial solution with initial distribution $\eta$.

TheOREM 3.9. Consider (2.1) in the case $0<\alpha<1$. Suppose that there exists a real number $\delta>1$ such that $|b|^{-\delta}$ is locally integrable. Moreover there exists $U>0$ such that $\lambda\left(B_{U}\right)<\infty$, where $B_{U}=\{x \in \mathbb{R}:|b(x)|>U\}$. Then, for any law $\eta$ on $\mathbb{R}$, there exists a nontrivial solution with initial distribution $\eta$.

Now we consider "local" solutions of (2.1).

We examine the subject at this stage because "local" existence results are proven using the above "global" existence ones. Let $A=] u, v[$ (resp. $A=[u, v]$ ) denote an open (resp. closed) interval of the real line with $-\infty \leq u<v \leq+\infty$ (resp. $-\infty<u<v<+\infty$ ) and $x$ a real number in $A$ (resp. in the interior of $A$ ). According to Definition (3.1) in [18], a process $(X, \mathbb{F})$, defined on a basis $(\Omega, \mathcal{F}, \mathbb{F}, P)$, is called $a$ solution of $(2.1)$ on the interval $A$ starting from $x$ if there exists an $\alpha$-stable Lévy process $(Z, \mathbb{F})$ such that $\int_{[0, t \wedge T]} b\left(X_{s^{-}}\right) d Z_{S}$ exists 
for every $t$, where $T=\inf \left\{s: X_{S} \notin A\right\}$ is the first exit time of $A$ for $X$ and such that, up to $P$-equality,

$$
X_{t \wedge T}=x+\int_{] 0, t \wedge T]} b\left(X_{s^{-}}\right) d Z_{s} \text { for all } t .
$$

In an obvious way we define triviality of a solution on an interval $A$ as above: see Definition (3.2) in [18], Section 3. Now note that all "local" results in [18] can be easily extended to the cases when (2.1) is driven by an $\alpha$-stable Lévy motion not necessarily symmetric (the coefficient $b$ being in the latter case nonnegative) including the case $\alpha=1$ : it suffices to apply Theorem 3.3 by using the same localizing methods as in [18].

The following theorem unifies the cases of (2.1) with different $\alpha, 0<\alpha \leq 2$.

THEOREM 3.10. Consider (2.1) driven by an $\alpha$-stable Lévy process $Z, 0<$ $\alpha \leq 2$. Assume that the coefficient $b$ satisfies condition (LH) with respect to a real number $x$. Then there exists a nontrivial solution on the interval $[x-\varepsilon, x+\varepsilon]$ starting from $x, \varepsilon$ denoting any number such that the relation defining $(\mathrm{LH})(x)$ is satisfied.

PROOF. The proof is similar to that of Theorem (3.4) in [18], to which the reader is referred.

By using the same integrability properties of $\alpha$-stable transition densities that lead to Proposition 3.7, from the last theorem we obtain the following.

PROPOSITION 3.11. (a) Consider the case $1<\alpha \leq 2$. Let $x \in \mathbb{R}$ be such that there exists an $\varepsilon>0$ with

$$
\int_{-\varepsilon}^{+\varepsilon} \frac{1}{|b(x+y)|^{\alpha}} d y<\infty .
$$

Then coefficient $b$ satisfies condition $(\mathrm{LH})(x)$ and there exists a nontrivial solution of (2.1) on the interval $[x-\varepsilon, x+\varepsilon]$ starting from $x$.

(b) Consider the case $0<\alpha \leq 1$. Let $x \in \mathbb{R}$ be such that there exist two real numbers $\varepsilon>0, \delta>1$ with

$$
\int_{-\varepsilon}^{+\varepsilon} \frac{1}{|b(x+y)|^{\delta}} d y<\infty .
$$

Then coefficient $b$ satisfies condition $(\mathrm{LH})(x)$ and there exists a nontrivial solution of (2.1) on the interval $[x-\varepsilon, x+\varepsilon]$ starting from $x$.

Also the following necessary condition for "local" existence holds, similar to Proposition 2.1. 
Proposition 3.12. Let A denote an open (when considering $] u, v[$, the case $u=-\infty$ or $v=+\infty$ is included; recall the definition of a solution on an interval) or compact nondegenerate interval and let $x$ be in the interior of A. Assume that $Z, X$ resp. denote an $\alpha$-stable Lévy motion $(0<\alpha \leq 2)$ and a process defined on a basis $\mathbb{B}=(\Omega, \mathcal{F}, \mathbb{F}, P)$ and solving (2.1) on $A$, with $X_{0}=x$ a.s. Moreover $X$ is nontrivial. Then the same conclusion as in Proposition 2.1 holds.

PROOF. Proceeding as in the proof of Proposition (3.13) in [18], we consider first the case of a solution on $A=] u, v$ [ and introduce the process $Y$, which is $X$ stopped at the first exit time of $A$. It is easy to verify that, for all $t, Y$ solves the same equation as (2.1) but with the coefficient $\tilde{b}$ that is defined as

$$
\tilde{b}(y)=b(y) \mathbb{1}_{A}(y) .
$$

Then, on account of Proposition 2.1, there exists a $t>0$ such that

$$
P\left(\left\{\int_{0}^{t} \frac{d s}{\left|\tilde{b}\left(Z_{s}^{\prime}\right)\right|^{\alpha}}<\infty\right\}\right)>0,
$$

where $Z^{\prime}=x+Z$. It follows that $P\left(\left\{\int_{0}^{\tau} \frac{d s}{\left|b\left(Z_{s}^{\prime}\right)\right|^{\alpha}}<\infty\right\}\right)>0$, where $\tau=t \wedge \tau^{\prime}, \tau^{\prime}$ denoting the first exit time of $A$ for process $Z^{\prime}$ : indeed, when $s<\tau^{\prime}, Z_{s}^{\prime}$ is in $A$ so that $\tilde{b}\left(Z_{s}^{\prime}\right)=b\left(Z_{s}^{\prime}\right)$. However, $\tau$ is a.s. strictly positive; thus there exists a $t^{\prime}>0$ with $P\left(\left\{\tau>t^{\prime}, \int_{0}^{\tau} \frac{d s}{\left|b\left(Z_{s}^{\prime}\right)\right|^{\alpha}}<\infty\right\}\right)>0$ so that $P\left(\left\{\int_{0}^{t^{\prime}} \frac{d s}{\left|b\left(Z_{s}^{\prime}\right)\right|^{\alpha}}<\infty\right\}\right)>0$.

In the case of a solution on a compact interval it suffices to use Lemma (3.12) in [18].

Because of the last proposition and the above-recalled integrability properties of stable transition densities, we have the following.

COROLlary 3.13. Consider (2.1) driven by $Z, 1<\alpha \leq 2$. Then condition $(\mathrm{LH})(x)$ for $b$ is necessary for the existence of a nontrivial "local" solution starting from $x$ (and so also for the existence of a nontrivial solution starting from $x)$.

Moreover, from Theorem 3.10 and Propositions 3.11 and 3.12 we also have the following.

COROLlaRY 3.14. Fix $\alpha, 1<\alpha \leq 2$. For $x \in \mathbb{R}$ the following properties are equivalent:

(a) Function b satisfies condition $(\mathrm{LH})(x)$.

(b) The number $x$ does not belong to the set I of (2.3).

By combining results in Section 2 with the latter ones we have the following. 
THEOREM 3.15. Consider (2.1) driven by an $\alpha$-stable Lévy process $Z, 1<$ $\alpha \leq 2$. Then the following statements are equivalent:

(a) There exists an $x_{0} \in \mathbb{R}$ such that b satisfies condition $(\mathrm{H})$ with respect to $x_{0}$.

(b) Coefficient $b$ satisfies condition $(\mathrm{H})$ with respect to any $x$.

(c) Coefficient $b$ satisfies condition $(\mathrm{LH})$ with respect to any $x$.

(d) The set I of (2.3) is empty.

(e) Function $|b|^{-\alpha}$ is locally integrable.

(f) For every $x$ there exists a nontrivial solution starting from $x$.

(g) For every $x$ there exists an open (or compact nondegenerate) interval $A_{x}$ containing $x$ in its interior and a nontrivial solution on $A_{x}$ starting from $x$.

(h) For every law $\eta$ on $\mathbb{R}$, there exists a nontrivial solution with initial law $\eta$.

Proof. Clearly (d) $\Leftrightarrow$ (e) and by Corollary 3.14 (c) $\Leftrightarrow$ (d). Now define process $C$ of (2.4) employing an $\alpha$-stable motion $Z$ starting from $x_{0}$. If (a) holds, as in the proof of Theorem 3.3, the case $\alpha$ with $1<\alpha<2$, we see that $I$ is empty; that is, (d) holds. Because of Proposition 3.7, (e) $\Rightarrow$ (b) and (b) $\Rightarrow$ (a), of course. Thus statements (a), (b), (c), (d), (e) are equivalent. Moreover (b) $\Rightarrow$ (f) by Theorem 3.3 and (f) $\Rightarrow(\mathrm{g})$ of course. But $(\mathrm{g}) \Rightarrow$ (c) by Corollary 3.13. Finally, (b) $\Rightarrow$ (h) because of Lemma 3.4, taking Remark 3.6 into account. But (h) $\Rightarrow$ (f) and we are done.

The following examples illustrate the previous results and comment on the conditions $(\mathrm{H})(x)$ and $(\mathrm{LH})(x)$.

EXAMPLE 3.16. Let us consider the following equation:

$$
X_{t}=x+\int_{10, t]} X_{s^{-}} d Z_{s}, \quad t \geq 0(x \in \mathbb{R}),
$$

driven by a symmetric $\alpha$-stable Lévy process $Z$, with $1<\alpha \leq 2$. In the present case coefficient $b, b(x)=x$ identically, is such that the singularity set $I$ of (2.3) for $|b|^{-\alpha}$ verifies relation $I=\{0\}=N$. So Theorem 2.6 holds and the equation has the a.s. null process, that is, the trivial solution, as the only one starting from 0 (on any base carrying a process $Z$ with the required law). Because of the last theorem, coefficient $b$ does not satisfy condition $(\mathrm{H})(x)$ for all $x$. However, for each $x \neq 0$, there exists the solution starting from $x$, which is constructed by time change as in the proof of Theorem 2.2 and such solution is nontrivial (cf. Remark 2.4). It follows that condition $\mathrm{H}(x)$ is not necessary for the existence of a nontrivial solution starting from $x$.

As to condition (LH), $b$ does not satisfy condition (LH)(0): if not, 0 would not be in $I$, as we see from Corollary 3.14. But $b$ satisfies (LH) $(x)$ for all $x \neq 0$, as follows from Corollary 3.13.

In general, it is well known that, when driven by a semimartingale, the above equation admits a unique strong solution (on any appropriate basis), which is given 
by the exponential formula [cf. [6], Theorem (6.2)]. So, also when $Z$ has parameter $\alpha$ with $0<\alpha \leq 1$, the equation has the a.s. null process as the only solution starting from 0 . But for every $x \neq 0$, there exists a nontrivial solution. Also, when $0<\alpha \leq 1$, coefficient $b$ does not satisfy condition (LH)(0): indeed, assume that (LH) (0) holds; that is, there exists an $\varepsilon>0$ such that $\int_{0}^{\varepsilon} d s\left(\int_{-\varepsilon}^{+\varepsilon} \frac{f(s, y)}{|y|^{\alpha}} d y\right)<+\infty$. Then, using Lemma (3.5) in [18], it is immediate to see that condition $(\mathrm{H})(0)$ is satisfied by the function $\tilde{b}$ defined as

$$
\tilde{b}(y)=-\varepsilon \mathbb{1}_{]-\infty,-\varepsilon]}(y)+y \mathbb{1}_{-\varepsilon, \varepsilon[}(y)+\varepsilon \mathbb{1}_{[\varepsilon,+\infty[}(y) .
$$

Then Theorem 3.3 implies that there exists a nontrivial solution of the equation $X_{t}=x+\int_{] 0, t]} \tilde{b}\left(X_{s^{-}}\right) d Z_{s}$ starting from 0 . However, the function $\tilde{b}$ being Lipschitz, the only solution of such an equation starting from 0 is the null process, that is, the trivial one, and we get a contradiction.

Since $b$ does not satisfy condition $(\mathrm{LH})(0)$, it does not satisfy condition $(\mathrm{H})(0)(0<\alpha \leq 1)$. On the other hand, $b$ satisfies condition $(\mathrm{LH})(x)$ for every $x \neq 0$, as one deduces from Proposition 3.11. We conclude that, for all $\alpha, 0<\alpha \leq$ 2 , coefficient $b$ satisfies condition (LH) $(x)$ for any $x \neq 0$ (i.e., any $x$ such that there exists a nontrivial solution starting from $x$ ) and does not satisfy (LH) $(0)$, the trivial one being the only solution starting from 0 .

EXAmple 3.17 (Due to H. Pragarauskas). Consider the equation

$$
X_{t}=x+\int_{] 0, t]}\left|X_{s^{-}}\right|^{1+\varepsilon} d Z_{s}, \quad t \geq 0(x \in \mathbb{R}),
$$

driven by a symmetric $\alpha$-stable Lévy motion $Z, \varepsilon$ being a constant $>0$ and $x \in \mathbb{R}$. When $1<\alpha \leq 2$, point 0 is in the singularity set $I$ from (2.3) of $|b|^{-\alpha}$, where $b$ is the coefficient, $b(x)=|x|^{1+\varepsilon}$ identically. Thus, starting with an $\alpha$-stable Lévy motion $Z, Z_{0}=0$, time $D$ of (2.6) is 0 and Lemma 2.3 implies, a.s.,

$$
C_{t}=\int_{0}^{t} \frac{d s}{\left|b\left(Z_{s}\right)\right|^{\alpha}}=\infty \quad \text { for all } t>0 .
$$

However, the last relation holds also in the case $0<\alpha \leq 1$. Indeed, for any Borel function $f \geq 0$ and any integer $n \geq 0$ one has

$$
\int_{0}^{t} f\left(Z_{s}\right) d s=\int_{\mathbb{R}} f(y) \sigma(t, d y) \geq \int_{\Gamma_{n}} f(y) \sigma(t, d y) \geq n \cdot \sigma\left(t, \Gamma_{n}\right),
$$

where $\Gamma_{n}=\{x: f(x)>n\}$ and $\sigma(t, \Gamma)=\int_{0}^{t} \mathbb{1}_{\Gamma}\left(Z_{s}\right) d s$ denotes the occupation time measure of $Z$. Note that in our case, with function $f=|b|^{-\alpha}$, for any $n, \Gamma_{n}=$ $S_{n^{-1 /(\alpha+\varepsilon \alpha)}}$, where $S_{r}=\{x:|x|<r\}$. Moreover, for all $r>0$, the distributions of $r^{\alpha} \sigma\left(t, S_{1}\right)$ and $\sigma\left(t r^{\alpha}, S_{r}\right)$ coincide. Indeed, owing to the self-similarity property 
of stable motions, for every $r>0$, process $\left(\frac{1}{r} Z_{r^{\alpha}}\right)_{s \geq 0}$ has the same law as $\left(Z_{s}\right)_{s \geq 0}$ and in particular we have

$$
\begin{aligned}
\sigma\left(t, S_{1}\right) & =\int_{0}^{t} \mathbb{1}_{S_{1}}\left(Z_{s}\right) d s \\
& \sim \int_{0}^{t} \mathbb{1}_{S_{1}}\left(\frac{1}{r} Z_{r^{\alpha} s}\right) d s \\
& =\int_{0}^{t r^{\alpha}} \mathbb{1}_{S_{1}}\left(\frac{1}{r} Z_{u}\right) \frac{d u}{r^{\alpha}}=\frac{1}{r^{\alpha}} \sigma\left(t r^{\alpha}, S_{r}\right),
\end{aligned}
$$

where $\sim$ denotes equality in law. Also, for all $n$,

$$
Y_{n}=n \cdot \sigma\left(t n^{-\alpha /(\alpha+\varepsilon \alpha)}, S_{n^{-1 /(\alpha+\varepsilon \alpha)}}\right) \sim n \cdot n^{-\alpha /(\alpha+\varepsilon \alpha)} \cdot \sigma\left(t, S_{1}\right)=X_{n} .
$$

Since $\sigma\left(t, S_{1}\right)$ is a.s. $>0$ owing to the right-continuity of $Z$, we have $\lim _{n \rightarrow \infty} X_{n}=\infty$ a.s.; thus $\lim _{n \rightarrow \infty} X_{n}=\infty$ in probability and, passing if necessary to a suitable subsequence (still denoted by $n$ ),

$$
\lim _{n \rightarrow \infty} Y_{n}=\infty \text { a.s. }
$$

Combining such a property with the fact that, for $n>1$,

$$
\sigma\left(t, \Gamma_{n}\right)=\sigma\left(t, S_{n^{-1 /(\alpha+\varepsilon \alpha)}}\right) \geq \sigma\left(t n^{-\alpha /(\alpha+\varepsilon \alpha)}, S_{\left.n^{-1 /(\alpha+\varepsilon \alpha)}\right)},\right.
$$

from (3.7) we get

$$
\int_{0}^{t} \frac{d s}{\left|b\left(Z_{s}\right)\right|^{\alpha}}=\infty \quad \text { a.s. }
$$

and finally (3.6), $C$ being increasing. So, from Proposition 3.12, it follows that the only solution starting from 0 is the a.s. null process, that is, the trivial solution (on any appropriate basis), for all $\alpha, 0<\alpha \leq 2$. For all $\alpha$, coefficient $b$ does not satisfy condition $(\mathrm{LH})(0)$ because of Theorem 3.10 and Proposition 3.12 and so $b$ does not satisfy condition $(\mathrm{H})(0)$. Note also that, for all $\delta>1$, point 0 is in the singularity set $I^{\delta}$ defined similarly to $I$ in (2.3) but with $\delta$ replacing $\alpha$ [and is not in this set when $(1+\varepsilon) \delta<1$, i.e., $\delta<\frac{1}{1+\varepsilon}$ ]. On the contrary, thanks to Proposition $3.11, b$ satisfies condition $(\mathrm{LH})(x)$ for every $x \neq 0$, so there exists a nontrivial local solution for every $x \neq 0$ thanks to Theorem 3.10 .

Acknowledgments. The author is very grateful to Prof. H. Pragarauskas for valuable discussions on the subject as well as to Prof. K. Sato for a personal communication which led to improvement of an earlier version of the paper.

This is an improved version of [20]. Results in the present paper were communicated at the 7th Vilnius Conference on Probability Theory and Mathematical Statistics, Vilnius (Lithuania), August 12-18, 1998. They were also the subject of an invited lecture at the Conference on Lévy Processes: Theory and Applications, Aarhus (Denmark), January 18-22, 1999 (cf. [21]). 


\section{REFERENCES}

[1] Bertoin, J. (1996). Lévy Processes. Cambridge Univ. Press.

[2] Dellacherie, C. (1971). Capacités et Processus Stochastiques. Springer, Berlin.

[3] Engelbert, H. J. and Schmidt, W. (1985). On solutions of one-dimensional stochastic differential equations without drift. Z. Wahrsch. Verw. Gebiete 68 287-314.

[4] Engelbert, H. J. and Schmidt, W. (1985). On one-dimensional stochastic differential equations with generalized drift. Lecture Notes in Control and Information Sciences 69 143-155. Springer, Berlin.

[5] Ikeda, N. and Watanabe, Sh. (1981). Stochastic Differential Equations and Diffusion Processes. North-Holland, Amsterdam.

[6] JACOD, J. (1979). Calcul Stochastique et Problèmes de Martingales. Lecture Notes in Math. 714. Springer, Berlin.

[7] JACOD, J. and Shiryaev, A. N. (1987). Limit Theorems for Stochastic Processes. Springer, Berlin.

[8] KallenberG, O. (1992). Some time change representations of stable integrals, via predictable transformations of local martingales. Stochastic Process. Appl. 40 199-223.

[9] Kallenberg, O. (1997). Foundations of Modern Probability. Springer, New York.

[10] Karatzas, I. and Shreve, S. E. (1994). Brownian Motion and Stochastic Calculus, 2nd ed. Springer, New York.

[11] Port, S. C. (1967). Hitting times for transient stable processes. Pacific J. Math. 21 161-165.

[12] Protter, P. (1990). Stochastic Integration and Differential Equations. Springer, Berlin.

[13] Revuz, D. and Yor, M. (1991). Continuous Martingales and Brownian Motion. Springer, Berlin.

[14] Samorodnitsky, G. and TAqQU, M. S. (1994). Stable Non-Gaussian Random ProcessesStochastic Models with Infinite Variance. Chapman and Hall, London.

[15] Sato, K. (1997). Time evolution of Lévy processes. In Trends in Probability and Related Analysis (N. Kono and N.-R. Shieh, eds.) 35-82. World Scientific, Singapore.

[16] Stone, CH. (1963). The set of zeros of a semistable process. Illinois J. Math. 7 631-637.

[17] TAYlor, S. J. (1967). Sample path properties of a transient stable process. J. Math. Mech. 16 $1229-1246$.

[18] Zanzotto, P. A. (1997). On solutions of one-dimensional stochastic differential equations driven by stable Lévy motion. Stochastic Process. Appl. 68 209-228.

[19] Zanzotto, P. A. (1998). Representation of a class of semimartingales as stable integrals. Teor. Veroyatnost. i Primenen. 43 808-818. (In English.)

[20] Zanzotto, P. A. (1998). On stochastic differential equations driven by Cauchy process and the other stable Lévy motions. Technical Report 2.312.1127, Dept. Mathematics, Univ. Pisa.

[21] Zanzotto, P. A. (1999). On stochastic differential equations driven by Cauchy process and the other $\alpha$-stable motions. In Mini-Proceedings: Conference on Lévy Processes: Theory and Applications 179-183. MaPhySto, Univ. Aarhus.

FACOLTÀ DI ECONOMIA

UNIVERSITÀ DI UDINE

VIA TOMADINI, 301A

33100 UDINE

ITALY

E-MAIL: zanzotto@dm.unipi.it 